\title{
Polycythemia Vera Presenting as Tinnitus and Hearing Loss: A Case Report
}

\author{
How Kit Thong ${ }^{1 *}$, Kim Yen Goh², Aminuddin Bin Saim ${ }^{1,3}$ \\ ${ }^{1}$ Department of Otorhinolaryngology, Head and Neck Surgery Faculty of Medicine, KPJ Healthcare University College, Negeri \\ Sembilan, Malaysia; ${ }^{2}$ Department of Clinical Haematology and Stem Cell Transplant, KPJ Ampang Puteri Specialist Hospital, \\ Selangor, Malaysia; ${ }^{3}$ Department of Otorhinolaryngology, Head and Neck Surgery, KPJ Ampang Puteri Specialist Hospital, \\ Selangor, Malaysia
}

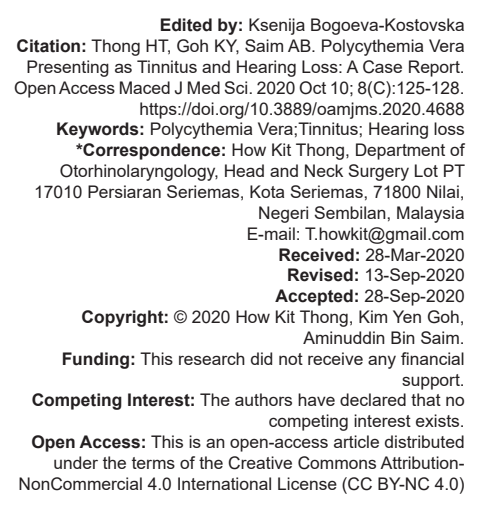

\section{Introduction}

Polycythemia vera (PV) is a chronic myeloproliferative neoplasm (MPN) characterized by erythrocytosis and commonly associated with the JAK2 V617F mutation. PV, essential thrombocythemia (ET), and primary myelofibrosis (PMF) belong to the same group of Philadelphia chromosome-negative MPNs [1]. PV is a relatively rare disease with a prevalence rate of 22 per 100,000 , and the incidence is higher in men than women. $\mathrm{PV}$ is typically diagnosed at 60-65 years of age [2]. The course of $\mathrm{PV}$ is variable as some patients are diagnosed only after routine blood work with few or no symptoms. In symptomatic patients, complaints are often non-specific; these symptoms include headaches, dizziness, blurring of vision, and pruritus. Severe complications are attributed to a high number of red blood cells and platelets that cause hyperviscosity and thrombotic events, such as cerebral infarctions, myocardial infarctions, and pulmonary embolisms [3]. Historically, hearing impairments were first described as a complication of PV in 1963, and it was stated that symptoms recovered after a phlebotomy. Occlusion and thrombosis of the feeding labyrinthine artery causing cochlear ischemia were thought to be the cause of such symptoms and complaints; however, tinnitus and hearing loss are rare initial presentations for patients with PV [4]. We are reporting a case of PV that was presented in our ENT outpatient clinic; the patient presented with a main complaint of sudden-onset bilateral tinnitus with onesided hearing loss.

\section{Case Report}

A 55-year-old gentleman presented with bilateral tinnitus and left-sided hearing loss that had been present for a month at our outpatient clinic. The symptoms occurred acutely and were not associated with otalgia, otorrhea, or dizziness. There was no previous history of acoustic trauma, otologic trauma, or drug intake. The patient denied any underlying chronic illnesses or comorbidities. His family history was negative for any hematological malignancies or hereditary hearing loss. On physical examination, both the external ear canal and tympanic membrane appeared normal. There was no facial nerve palsy or ophthalmoplegia. A nasal endoscopy was performed, nasopharynx and bilateral eustachian tube were found 
to be normal. The tuning fork test showed a bilateral positive Rinne's test with no lateralization on Weber's test. Other systemic examinations were unremarkable.

Investigations showed a hemoglobin of $20.5 \mathrm{~g} / \mathrm{dL}$, a packed cell volume of $61 \%$, platelet counts of $40310^{3}$, $\mathrm{uL}$, a total white blood cell count of $11.110^{3} / \mathrm{uL}$, and an erythrocyte sedimentation rate of $1 \mathrm{~mm} / \mathrm{hr}$. Pure tone audiometry showed normal hearing in the right ear and mild to moderately severe sensorineural hearing loss with downsloping over a high frequency with an average hearing level of $45 \mathrm{db}$ (Figure 1). Tympanometry was performed and revealed Type A in both ears, which signifies normal middle ear pressure and function. Magnetic resonance imaging (MRI) of the brain and cerebellopontine angle was also performed which was reported as normal with no evidence of cerebellopontine angle mass.

The patient was subsequently referred to the department of hematology and oncology. Further investigation revealed erythrocytosis and thrombocytosis on peripheral blood smears and a decreased level of erythropoietin $3.0 \mathrm{mU} / \mathrm{mL}$. The patient was tested positive for the JAK2 V6617F mutation. Based on two major criteria (hemoglobin $20.5 \mathrm{~g} / \mathrm{dL}$ and the patient being JAK V6617F mutation positive) with one minor criteria (erythropoietin level of $3 \mathrm{mU} / \mathrm{L}$ ), a diagnosis of PV was made. The patient was started on $500 \mathrm{mg}$ of hydroxyurea twice daily, $100 \mathrm{mg}$ of aspirin daily, and a phlebotomy was performed 3 times over a period of 6 months. After 5 months of treatment, a review of the blood analysis was as follows: Hemoglobin was at $15.8 \mathrm{~g} / \mathrm{dL}$, hematocrit was $48 \%$, and the platelet count was $305,000 /$ $\mathrm{mcL}$. A repeated audiogram noted improvement in the left ear hearing at the higher frequency (Figure 2). The patient also reported a reduction in the intensity of the tinnitus. Tinnitus was also subjectively measured using the tinnitus handicap inventory score. On presentation, the score was 74/100; the second score was $68 / 100$ after 9 weeks of treatment when the hemoglobin level was $15.4 \mathrm{~g} / \mathrm{dL}$ with a packed cell volume of $45 \%$. The
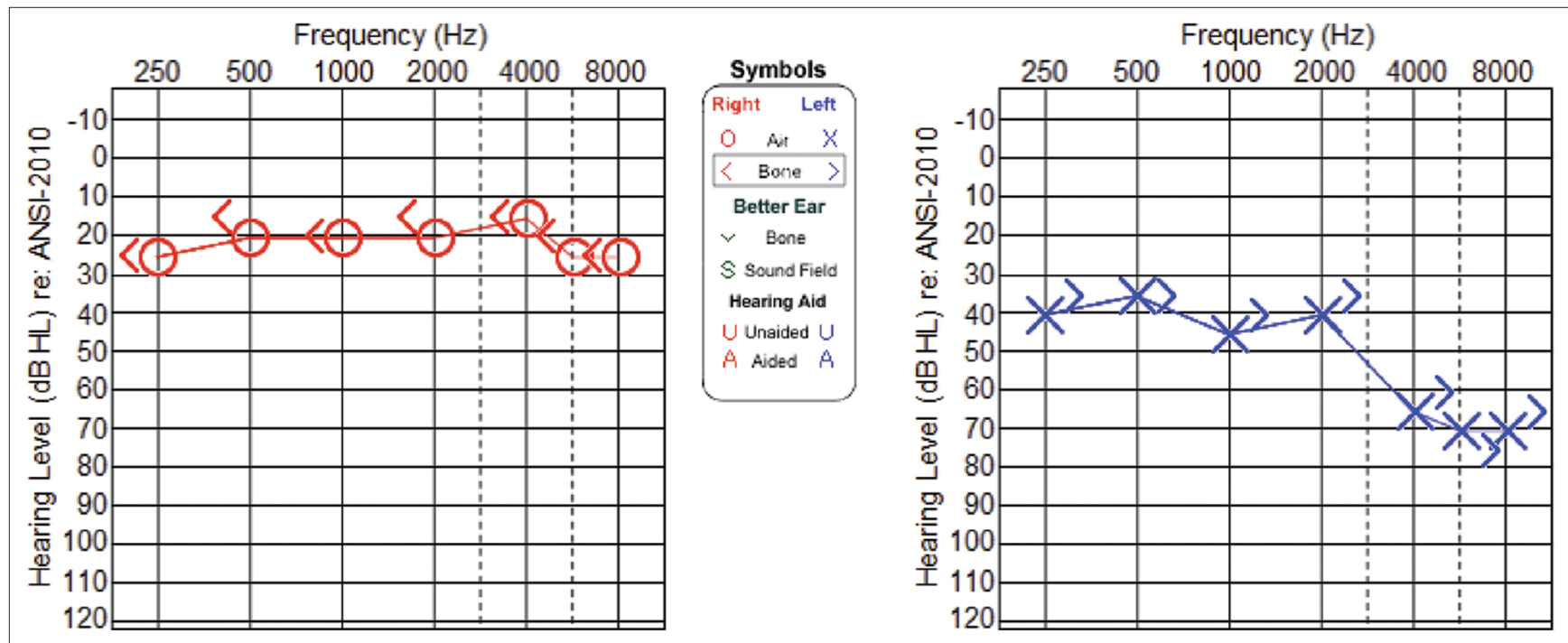

Figure 1: Pure tone audiometry on the first visit
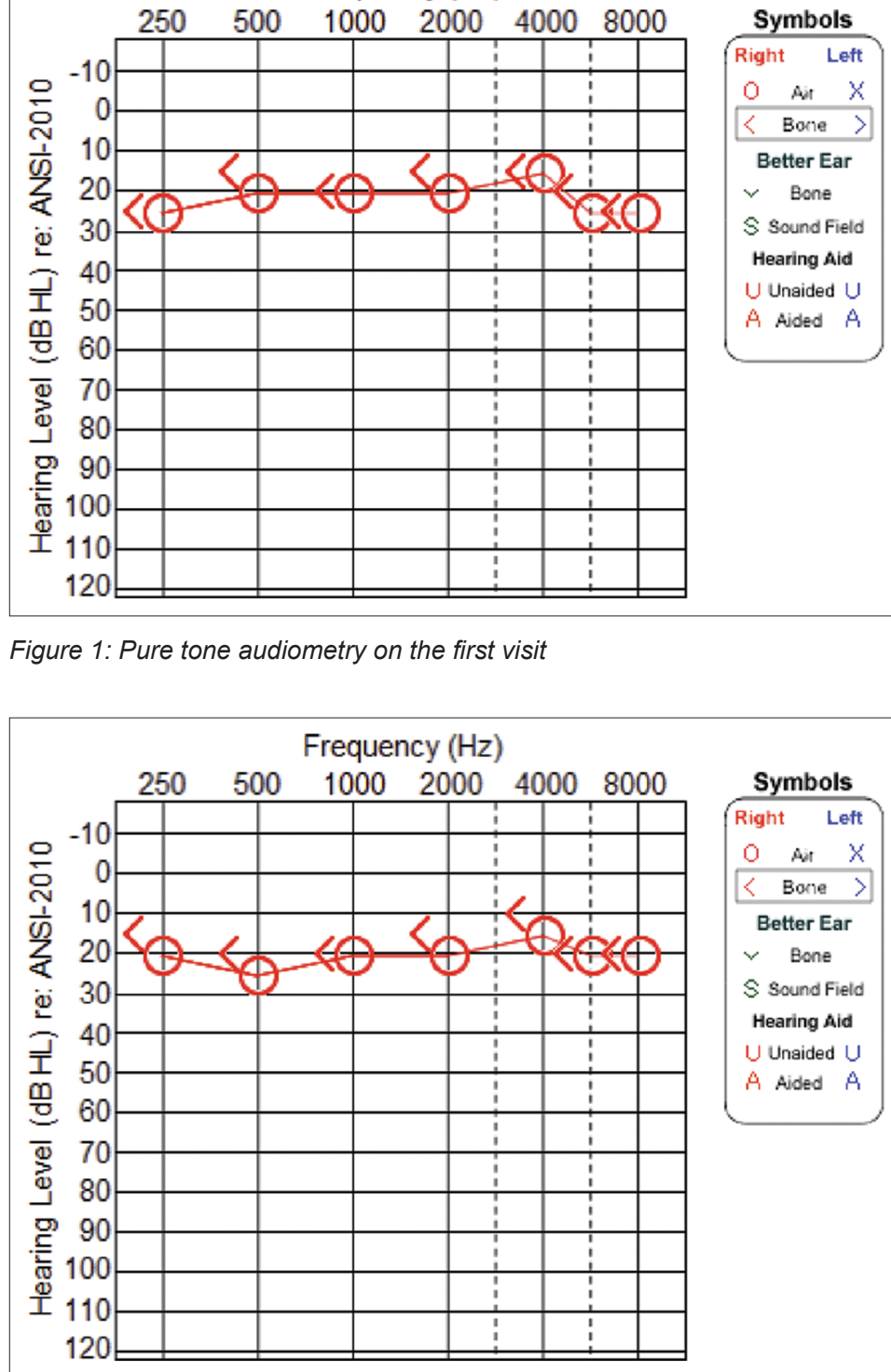

Figure 2: Pure tone audiometry on post-treatment 
patient was followed up on regularly by our team and the hematology department. Ginkgo biloba at a $150 \mathrm{mg}$ dosage twice daily was subsequently initiated, and the patient reported improvements in the intensity of tinnitus with a score of 58/100.

\section{Discussion}

The classification for hematopoietic neoplasm was recently revised in 2016. It recognizes MPN as a part of the myeloid malignancies. MPN includes PV, ET, and PMF [5]. PV is the most common among the group of MPN with an incidence of $0.01-2.61 / 100,000$ and a prevalence of $0.49-46.88 / 100,000$. The 5-year relative survival of PV was reported to be as high as $84.8 \%$ [6]. The diagnosis of $\mathrm{PV}$ is based on the revised World Health Organization criteria of three major or the first major and one minor criteria. The major criteria include (1) hemoglobin $>16.5 \mathrm{~g} /$ $\mathrm{dL}$ in men and $>16.0 \mathrm{~g} / \mathrm{dL}$ in women or hematocrit $>49 \%$ in men and $>48 \%$ women or increased red blood cell mass, (2) hypercellular bone marrow with trilineage growth, and (3) positive JAK2 mutation. The minor criterion is subnormal serum erythropoietin [5]. Common clinical symptoms range from mild non-specific complaints of pruritus, headache, weakness, and dizziness to severe complications of thrombosis and hemorrhaging. The reported incidence of thrombotic complications such as stroke, acute myocardial infarction, and pulmonary embolism in PV is between 13 and 60\%. Hemorrhagic complications include epistaxis, gastrointestinal hemorrhage, or ecchymosis. ENT symptoms of tinnitus, hearing loss, vertigo, and facial weakness are also associated with the disease. Hearing loss and tinnitus are the most commonly reported otological symptoms [7], [8]. Outcomes of such symptoms vary with some patients experiencing significant improvement [9]. The pathophysiology of hearing loss in patients with PV is caused by an elevated hematocrit, increased red blood cell mass, high viscosity, and increased acute-phase reactants causing thrombosis in small vessels such as the labyrinthine artery. Both thrombosis and increased viscosity cause microvascular occlusion and were accepted as the primary cause of symptoms in PV. In a study by Dogan et al., patients with known PV were compared to healthy controls. Cochlear impairment was found to be significantly more common in PV group ( $p=0.004)$ [10].

Kim et al. reported a case of $\mathrm{PV}$ which presented with symptoms of vertigo and on physical examination demonstrated gaze-evoked nystagmus. Similarly seen in our case, the findings and symptoms disappeared on normalizing of the blood parameters with repetitive phlebotomy and medical therapy of hydroxyurea and aspirin. The author also postulated that the pathophysiology of vertigo in PV could be explained by central vestibulopathy due to vascular insufficiency rather than peripheral vestibulopathy caused by inner ear vascular hyperviscosity [11].
However, hearing loss and tinnitus as initial reporting symptoms are rare, and no cases were found during a search of the PubMed archives. Before the conclusion of PV associated hearing loss in our patient, other organic causes of asymmetrical sensorineural hearing loss including acoustic neuroma were ruled out. PV is a chronic incurable disease with a reasonably good 5-year survival rate of $85 \%$. However, a detailed and thorough workup is needed to reduce the rate of misdiagnosis due to the conflation of patients with ET and PV. Phlebotomy is the primary treatment for PV. It can effectively lower the hematocrit to $<45 \%$ in males and $<42 \%$ in females, which will effectively reduce the risk of developing $\mathrm{PV}$ complications of thrombosis [1].

$A$ recently published guideline on the management of PV by Tefferi et al. proposed a risk stratification system in the treatment approach of patients with $\mathrm{PV}$. The main objective in the treatment of both patients with PV and ET is prevention of potential thrombohemorrhagic complications. All patients with $\mathrm{PV}$ are advised for phlebotomy to maintain the hematocrit level below $45 \%$ and once or twice daily aspirin. In the very low-risk ET/PV group, patients might not require therapy while aspirin therapy is advised for those with low-risk disease. Cytoreductive therapy, however, is recommended only for high-risk ET and PV. The drug of choice for cytoreductive therapy, in both ET and $\mathrm{PV}$, is hydroxyurea and second-line drugs of choice are interferon- $\alpha$ and busulfan [12]. For our patient, phlebotomy was performed 3 times during the initial 6 months of therapy. The patient was also started on longterm hydroxyurea therapy and aspirin for prophylaxis of thrombosis with phlebotomy performed periodically to keep hematocrit $<45 \%$. The duration of treatment will be determined by the hemato-oncology team based on close monitoring of symptoms and clinical response.

Ruxolitinib, a JAK 1 and JAK 2 selective inhibitor, is still being evaluated as a treatment option in view of the high rate of JAK2 mutations in patients with PV. At present, ruxolitinib has been approved by the US Food and Drug Administration for the treatment of patients with intermediate- and high-risk myelofibrosis or those with an inadequate response or are intolerant of hydroxyurea [11]. For our patient, after the phlebotomy was performed, the laboratory values of hemoglobin and hematocrit improved to normal limits. The patient noted that there were improvements in the tinnitus; however, his hearing did not recover to normal even though significant improvements were noted on the repeated pure tone audiometric tests post-treatment. A longer duration of follow-up is needed to determine the long-term outcome of $\mathrm{PV}$ on hearing loss and tinnitus in the patient.

\section{Conclusion}

We presented a case of a newly diagnosed PV patient with an unusual initial presentation of 
tinnitus and hearing loss. This case emphasizes the importance of otolaryngologists remaining vigilant and maintaining a high index of suspicion as $\mathrm{PV}$ can present with audiological symptoms. The early diagnosis and initiation of treatment can improve patients' hearing and reduce the morbidity of such patients.

\section{References}

1. Spivak JL. Polycythemia vera. Curr Treat Options Oncol. 2018;19(2):12.

PMid:29516275

2. Landolfi R, Nicolazzi MA, Porfidia A, Di Gennaro L. Polycythemia vera. Intern Emerg Med. 2010;5(5):375-84. https://doi. org/10.1007/s11739-010-0369-6

PMid:20237866

3. Raedler LA. Diagnosis and management of polycythemia vera. Am Health Drug Benefits. 2014;7(7):S36-47.

PMid:26568781

4. Nilo ER, Davis EC. Hearing loss in polycythemia vera. J Speech Hear Disord. 1965;30:234-42. https://doi.org/10.1044/ jshd.3003.234

PMid:14321497

5. Arber DA, Orazi A, Hasserjian R, Thiele J, Borowitz MJ, Le Beau MM, et al. The 2016 revision to the World Health Organization classification of myeloid neoplasms and acute leukemia. Blood. 2016;127(20):2391-405. https://doi. org/10.1182/blood-2016-06-721662
PMid:27069254

6. Anderson LA, McMullin MF. Epidemiology of MPN: What do we know? Curr Hematol Malig Rep. 2014;9(4):340-9. https://doi. org/10.1007/s11899-014-0228-z

PMid:25129052

7. Cass ND, Gubbels SP, Portnuff CD. Sudden bilateral hearing loss, tinnitus, and vertigo as presenting symptoms of chronic myeloid leukemia. Ann Otol Rhinol Laryngol. 2018;127(10):731-4. https://doi.org/10.1177/0003489418787831

PMid:30032641

8. Chang $\mathrm{KH}$, Jeon EJ. Sensorineural hearing loss and tinnitus as presenting symptoms of polycythemia vera. J Int Adv Otol. 2012;8:317-20.

9. Chae SW, Cho JH, Lee JH, Kang HJ, Hwang SJ. Sudden hearing loss in chronic myelogenous leukaemia implicating the hyperviscosity syndrome. J Laryngol Otol. 2002;116(4):291-3. https://doi.org/10.1258/0022215021910564

PMid:11945192

10. Dogan EE, Uslu M, Ozkan MC, Unal HD, Saydam G, Ogut MF, et al. The effect of polycythemia vera on hearing functions: Evaluation of twenty-one patients. Clin Otolaryngol. 2019;44(3):434-7. https://doi.org/10.1111/coa.13303 PMid:30735002

11. Kim Y, Song I, Jeong S, Lee A, Kim J. Gaze-evoked and perverted head-shaking nystagmus in a patient with polycythemia vera. Res Vestib Sci. 2017;16(4):142-6. https://doi.org/10.21790/ rvs.2017.16.4.142

12. Tefferi A, Barbui T. Polycythemia vera and essential thrombocythemia: 2019 update on diagnosis, risk-stratification and management. Am J Hematol. 2018;94(1):133-43. https:// doi.org/10.1002/ajh.25303

PMid:30281843 